\title{
How much radiologically isolated syndrome suggestive of multiple sclerosis is multiple sclerosis?
}

\author{
O quanto a síndrome radiológica isolada sugestiva de esclerose múltipla é realmente \\ esclerose múltipla?
}

Marco Aurélio Lana-Peixoto

MD, PhD CIEM MS Research Center, Medical School of the Federal University of Minas Gerais, Belo Horizonte MG, Brazil.

Correspondence: Marco A. Lana-Peixoto

Rua Padre Rolim, 769 - Sala 1.301; 30130-090 - Belo Horizonte MG-Brasil E-mail:marco.lanapeixoto@gmail.com Conflict of interest:

There is no conflict of interest to declare.

Received 07 October 2011

Received in final form 14 October 2011
I n spite of the progress in Neuroimmunology, a specific biomarker for the diagnosis of multiple sclerosis (MS) remains to be defined. Meanwhile, as growing enthusiasm has been associated with the development of new therapeutic options and the recent evidence that the disease-modifying agents may be more beneficial for MS patients if initiated as early as possible following disease onset ${ }^{1}$, investigators and clinicians alike continuously search for early signs of the disease.

The incorporation of specific magnetic resonance imaging (MRI) features for dissemination in space and in time ${ }^{2,3}$ into the diagnostic criteria for MS by the International Panel ${ }^{4}$ and their revisions in $2005^{5}$ and $2010^{6}$ have allowed the earlier diagnosis of MS in patients, who still lack complete clinical evidence of dissemination in both space and time. However, the occurrence of symptoms consistent with neurologic dysfunction of the central nervous system still lingers as a basic requirement for its diagnosis. Additionally, as long as a specific biomarker is not introduced into clinical practice, alternative diagnoses must be excluded.

The diagnosis of MS is particularly challenging in a group of patients who present no history of symptoms consistent with MS or objective signs of neurologic dysfunction, whose brain MRI shows abnormal signals with spatial dissemination, which is highly suggestive of demyelinating disease. Such findings have been described in first-degree relatives of patients with MS who are at particular risk ${ }^{7}$, in some subjects with history of psychiatric disorders ${ }^{8}$, and surprisingly in other patients who undergo MRI scans for reasons that were not related to the MS investigation. The term radiologically isolated syndrome (RIS) was recently introduced to describe these individuals?.

In this Issue of Arquivos de Neuro-Psiquiatria, Maia Júnior et al. ${ }^{10}$ report their observations on the clinical and imaging features of 12 Brazilian patients with RIS during a median follow-up period of 49 months. Reasons for acquisition of brain MRI in their cohort as in other published series varied widely. The most frequent reasons for the first MRI scan in RIS patients in the reported series included migraine and other types of headache; trauma and radiculalgia; depression; epilepsy; endocrinopathies; and cognitive dysfunction. One half of the patients in the Brazilian cohort showed temporospatial dissemination of the MRI lesions on a second MRI scan during the follow-up period, whereas two subjects developed symptoms of neurologic dysfunction, as a clinically isolated syndrome (CIS), or recurring symptoms compatible with the diagnosis of clinically definite MS (CDMS).

Similar features of the Brazilian series had already been demonstrated in a French cohort in the initial description of RIS, in $2008^{11}$, and in subsequent published series ${ }^{9,12,13}$. In the published cohorts, during the follow-up period, many patients not only developed an increased number of T2-lesions on MRI, an evidence of temporospatial dissemination - and therefore MS by MRI criteria, but they also exhibited clinical manifestations of central nervous system dysfunction. This clinical eloquence could either take the form of CIS or CDMS. Additionally, patients with RIS, CIS or CDMS have a number of common epidemiological and imaging features, which differ from healthy controls. 
The more the RIS patients have paraclinical abnormalities, such as gadolinium enhancement in brain MRI and presence of oligoclonal bands or increased IgG index on the cerebrospinal fluid (CSF), the earlier the first neurological symptom occurs ${ }^{9,12,13}$. Patients who also develop increased number of MRI lesions during the follow-up period are also at higher risk of developing $\mathrm{CDMS}^{9,12,13}$. In a study of 70 RIS subjects during a mean follow-up period of 5.2 years, it was observed that $91 \%$ of them developed new MRI lesions, 37\% of which with gadolinium enhancement ${ }^{12}$. A clinical event occurred in $33 \%$ of the individuals, with the same clinical expression as classically described in CIS $^{14}$ in a mean time of 2.3 years. Eight patients showed progression to CDMS, but no one had progressive $\mathrm{MS}^{12}$.

Like unanticipated brain spatial dissemination of MRI lesions in the absence of clinical symptoms consistent with MS, MRI abnormal signals in the spinal cord in asymptomatic individuals are occasionally found, either following the finding of unanticipated brain MRI, or during orthopedic or medical workup. In a recent paper, Okuda et al. ${ }^{15}$ reported their findings on 71 RIS patients who had cervical spine MRI scans acquired prior to the development of the first clinical event. Cervical spinal cord abnormal signals highly suggestive of MS were disclosed in 25 patients (35\%), whereas clinical progression to CIS or primary progressive MS was observed in 21 (84\%) of the 25 subjects over a median time of 1.6 years. Only three patients out of 46, who did not have spinal cord lesions, developed a clinical event. The diagnostic predictive value of an asymptomatic spinal cord lesion in subjects with RIS for development of CIS or PPMS had a sensitivity of $87.5 \%$, specificity of $91.5 \%$ and positive predictive value of $84 \%$. These observations support the view that asymptomatic subjects who possess MRI abnormal signals in both brain and cervical spinal cord, in comparison with the brain alone, are at high risk for developing clinical $\mathrm{MS}^{15}$.

ManyauthorsconsiderRISasaformof"pre-clinicalMS”,11-13. Notwithstanding, most RIS subjects develop new MRI lesions in the course of time - dissemination in space and time. Some subjects still remain free from neurological symptoms to have their brain lesions only uncovered at the autopsy ${ }^{16}$. However, if one takes into account cognitive function appropriately assessed by a neuropsychological battery, the proportion of RIS subjects with silent lesions may be much lower. Cognitive impairment has been found in up to $70 \%$ in MS, it is present in patients in early stages of disease, it is only mildly associated with functional status as measured by the expanded disability status scale, and may be even identified in patients with $\mathrm{CIS}^{17}$. It affects several aspects of the higher cortical functioning including attention, information processing efficiency, executive functioning, processing speed, and longterm memory, although processing speed and visual learning and memory are most frequently impaired ${ }^{17}$. In this line of evidence, a recent paper ${ }^{18}$ showed that out of 26 RIS subjects who underwent neuropsychological evaluation none had a strictly normal cognitive function. When compared with healthy controls, the speed of information processing, executive functions and short-term memory were the most consistently cognitive functions impaired in RIS and MS patients. Differences between RIS and MS patients were seen only in the difficulty of performing executive tasks and in the shortterm memory. No correlation was found between sites and number of MRI lesions using Barkhof-Tintore criteria and cognitive dysfunction ${ }^{18}$. Other investigators have found that T1 hypointense and T2 hyperintense lesion volumes in MS patients are predictive factors for impaired performance in at least one cognitive test ${ }^{19}$.

As a result of the cognition impairment, RIS subjects may present fewer social and vocational activities, higher rate of unemployment, and greater difficulties in performing routine household tasks, being still more vulnerable to psychiatric illness than individuals with a purely physical disability ${ }^{20}$. Likewise, as cognition impairment has a direct impact on the quality of life (QoL), which is decreased in MS patients ${ }^{21}$, it is expected that future studies will demonstrate decreased QoL in subjects with RIS. However, neuropsychological assessment may depict high frequency of cognitive impairment with deficit of attention and concentration, impairment of executive functions, decreased speed of information processing, and involvement of short-term memory ${ }^{22}$. These cognitive changes are similar to those found in MS patients, but RIS subjects are usually less severely affected than MS ones ${ }^{18}$. This finding is in agreement with the early occurrence of cognitive impairment in MS and even its observation in CIS patients ${ }^{23,24}$.

Although RIS and MS patients have striking similarities on their conventional MRI features, the use of quantitative MR indices has provided evidences of their differences, as well as an explanation for the lack of clinical expression in RIS subjects ${ }^{25}$. Whereas other MRI metrics have shown that RIS and RRMS patients have values of global and tissue-specific volumes, which are similarly lower than those found in healthy controls, magnetization transfer ratio (MTr) imaging that provides information on tissue integrity at the cellular level, is much milder in RIS than in RRMS. MTr values in the normal-appearing brain were similar in RIS subjects and in healthy controls, but they were much higher than those in RRMS patients. Interestingly enough it was found that the areas with lower MTr in EMRR subjects as compared with RIS were the very eloquent ones, as they are usually deeply involved in pathological substrate of the disease. It is probable that RIS subjects may have a milder degree of demyelination or a better repair response to insult ${ }^{25}$. By using MRI metrics, the authors ${ }^{25}$ demonstrated the probability of 13/19 (70\%) RIS patients of being RRMS. Interestingly predictive factors included abnormal CSF (10/10 subjects), dissemination in time in a new MRI 
scan (9/10 subjects) and a positive cervical spine MRI (6/7 subjects).

As Maia Junior et al. ${ }^{10}$ emphasize in their analysis of the Brazilian series, "all patients with RIS should be considered to have high risk of developing MS". However, neurologists must take into consideration all predictive factors of conversion of
RIS in clinical MS, as well as the information provided by appropriate imaging techniques to grade this risk. Certainly in the near future, the introduction of both, more sharpened imaging techniques, and more efficacious and safer drugs will allow neurologists to make better judgments on starting therapy much earlier on these patients.

\section{References}

1. Comi G. Shifting the paradigm toward the earlier treatment of multiple sclerosis with interferon beta. Clin Ther 2009;31:1142-1157.

2. Barkhof F, Filippi M, Miller DH, et al. Comparison of MRI criteria at first presentation to predict conversion to clinically definite multiple sclerosis. Brain 1997;120:2059-2069.

3. Tintore M, Rovira A, Martinez MJ, et al. Isolated demyelinating syndrome: comparison of different MRI criteria to predict conversion to clinically definite multiple sclerosis. Am J Neuroradiol 2000; 21:702-706.

4. McDonald WI, Compston A, Edan G, et al. Recommended diagnostic criteria for multiple sclerosis: guidelines from the International Panel on the Diagnosis of Multiple Sclerosis. Ann Neurol 2001;50:121-127.

5. Polman CH, Reingold SC, Edan G, et al. Diagnostic criteria for multiple sclerosis: 2005 revisions to the "McDonald Criteria". Ann Neurol 2005;58:840-846.

6. Polman $\mathrm{CH}$, Reingold SC, Banwell B, et al. Diagnostic criteria for multiple sclerosis: 2010 revisions to the McDonald criteria. Ann Neurol 2011;69:291-302

7. De Stefano N, Cocco E, Lai M, et al. Imaging brain damage in firstdegree relatives of sporadic and familial multiple sclerosis. Ann Neurol 2006;59:634-639.

8. LyoolK, Seol HY, Byun HS, Renshaw PF. Unsuspected multiple sclerosis in patients with psychiatric disorders: a magnetic resonance imaging study. J Neuropsychiatry Clin Neurosci 1996;8:54-59.

9. Okuda DT, Mowry EM, Beheshtian A, et al. Incidental MRI anomalies suggestive of multiple sclerosis: the radiologically isolated syndrome. Neurology 2009;72:800-805.

10. Maia Junior AC, Rocha JA, Barros BR, Tilbery CP. Incidental demyelinating inflammatory lesions in asymptomatic patients: a Brazilian cohort with radiologically isolated syndrome and a critical review of current literature. Arq Neuropsiquiatr 2012;70:5-11.

11. Lebrun C, Bensa C, Debouverie M, et al. Unexpected multiple sclerosis: follow-up of 30 patients with magnetic resonance imaging, clinical conversion profile.J Neurol Neurosurg Psychiatry 2008;79:195-198.

12. Lebrun C, Bensa C, Debouverie M, et al. for CFSEP. Club Francophone de la Sclérose en Plaques. Radiologically isolated syndrome and magnetic resonance imaging, cerebrospinal fluid, and visual evoked potential: follow-up of 70 patients. Arch Neurol 2009;66:841-846.
13. Siva A, Saip S, Altintas A, et al. Multiple sclerosis risk in radiologically uncovered asymptomatic possible inflammatory demyelinating disease. Mult Scler 2009;15:918-927.

14. Miller D, Barkhof F, Montalban X, Thompson A, Filippi M. Clinically isolated syndromes suggestive of multiple sclerosis, part I: natural history, pathogenesis,diagnosis, and prognosis. Lancet Neurol 2005; $4: 281-288$

15. Okuda DT, Mowry EM, Cree BAC, et al. Asymptomatic spinal cord lesions predict disease progression in radiologically isolated syndrome. Neurology 2011;76:686-692.

16. Engell T. A clinical patho-anatomical study of clinically silent multiple sclerosis. Acta Neurol Scand 1989;79:428-430.

17. Rao SM, Leo GJ, Ellington L, Nauertz T, Bernardin L, Unverzagt F. Cognitive dysfunction in multiple sclerosis. II. Impact on employment and social functioning. Neurology 1991;41:692-696.

18. Lebrun C, Blanc F, Brassat D, Zephir H, de Seze J. Cognitive function in radiologically isolated syndrome. Mult Scler 2011;16:919-925.

19. Patti F, Amato MP, Trojano M, et al. for the COGIMUS Study Group. Cognitive impairment and its relation with disease measures in mildly disabled patients with relapsing-remitting multiple sclerosis: baseline results from the Cognitive Impairment in Multiple Sclerosis (COGIMUS) study. Mult Scler 2009;15:779-788.

20. Rao S, Leo G, Bernardin L, Unverzagt F. Cognitive dysfunction in multiple sclerosis: frequency, patterns, and predictions. Neurology 1991;41:685-691.

21. Cutajar R, Ferriani E, Scandellari C, et al. Cognitive function and quality of life in multiple sclerosis patients. J Neurovirol 2000;6 (Suppl): S186-S190.

22. Hakiki B, Goretti B, Portaccio E, Zipoli V, Amato MP. Subclinical MS: follow-up of four cases. Eur J Neurol 2008;15:858-861.

23. Schulz D, Kopp B, Kunkel A, Faiss JH. Cognition in the early stage of multiple sclerosis. J Neurol 2006;253:1002-1010.

24. Zipoli V, Goretti B, Hakiki B, et al. Cognitive impairment predicts conversion to multiple sclerosis in clinically isolated syndrome. Mult Scler 2010;16:62-67.

25. DeStefano N,Stromillo ML, Rossi F,etal.Improving the characterization of radiologically isolated syndrome suggestive of multiple sclerosis. PLoS ONE 2011;6:e19452. doi:10.1371/journal.pone.0019452. 